\title{
AVALIAÇÃO DO DESPERDÍCIO ALIMENTAR DAREFEIÇÃO ALMOÇO EM DUAS ESCOLAS PÚBLICAS DO DISTRITO DE AVEIRO
}

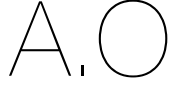

ARTIGO ORIGINAL

1 Faculdade de Ciências da Nutrição e Alimentação da Universidade do Porto, Rua do Campo Alegre, n. ${ }^{\circ} 823$,

4150-180 Porto, Portugal

2 Nutricionista, Especialista em Alimentação Coletiva e Restauração

${ }^{3}$ EPIUnit - Instituto de Saúde Pública da Universidade do Porto, Rua das Taipas, $n .{ }^{\circ} 135$ 4050-091 Porto, Portugal

"Endereço para correspondência:

Beatriz Ferreira Silva

Rua Padre Pereira da Costa,

n. 832 ,

3720-706, S. Roque - OAZ

Aveiro, Portugal

beaferreirasilva@gmail.com

Histórico do artigo:

Recebido a 10 de julho de 2020 Aceite a 18 de novembro de 2020

\section{EVALUATION OF FOOD WASTE FROM SCHOOL LUNCH IN TWO PUBLIC SCHOOLS IN THE DISTRICT OF AVEIRO}

Beatriz Ferreira Silva ${ }^{1 *}$; Beatriz Teixeira'; Helena Ávila²; Cláudia Afonso ${ }^{1,3}$

RESUMO

INTRODUÇÃo: O desperdício alimentar é uma problemática atual em refeitórios escolares. Numa lógica de sustentabilidade, torna-se essencial auscultar eventuais causas para, posteriormente, se proceder à sua redução. Para tal, a quantificação do desperdício alimentar é uma etapa fundamental.

OBJETIvos: Avaliar o desperdício alimentar dos alunos, na refeição do almoço, em duas unidades de restauração coletiva do setor escolar público.

METODOLOGIA: Estudo observacional de desenho transversal. Procedeu-se à avaliação do desperdício alimentar do item da refeição prato das refeições servidas a alunos do pré-escolar ao ensino secundário, durante 10 dias. Para quantificar as sobras e restos de 2686 refeições, considerou-se o método físico de pesagem.

RESULTADOS: Verificou-se 25\% de desperdício alimentar global para 2686 refeições servidas, observando-se um índice de sobra de produção e um índice de sobra de resto de, em média, 8\% e 18\%, respetivamente. Poderiam ser alimentados com o desperdício alimentar obtido 904 alunos.

CONCLUSÕES: O índice de sobra e o índice de sobra de resto nas componentes do prato apresentam-se acima dos valores de desperdício recomendados pela literatura ( $<3 \%$ índice de sobra e $<10 \%$ índice de sobra de resto), justificando uma urgente necessidade de intervenção.

\section{PALAVRAS-CHAVE}

Alimentação coletiva, Almoço escolar, Desperdício alimentar, Método físico de pesagem

ABSTRACT

INTRODUCTION: Food waste is an actual problem in school canteens. In a sustainable logic, it's essential to find out possible causes to reduce this issue. To this end, food waste quantification is fundamental.

OBJECTIVES: Evaluate the food waste of the main dish students, in the lunch meal, in two units of contract catering of the publicschool sector.

METHODOLOGY: Cross-sectional observational study. Evaluation of the food waste of the main dish was proceeded from preschoo to high school, during 10 days. In order to quantify the leftovers and plate waste of 2686 meals, the physical method was used. RESULTS: A ratio of $25 \%$ of overall food waste was verified for 2686 served meals, showing, in average, an index of leftovers and plate waste of $8 \%$ and $18 \%$, respectively. 904 students could be fed with the food waste obtained.

CONCLUSIONS: Leftovers and plate waste in the plate components, are above the waste values recommended by literature $(<3 \%$ and $<10 \%$, respectively), justifying an urgent need for intervention.

KEYWORDS

Contract catering, School lunch, Food waste, Physical method

\section{INTRODUÇÃO}

O Parlamento Europeu define desperdício alimentar (DA), como "o conjunto dos produtos alimentares que são eliminados da cadeia agroalimentar por razões económicas ou estéticas ou devido à proximidade do fim do prazo de consumo, mas que estão ainda em estado próprio para consumo humano e que, na ausência de um possível uso alternativo, se destinam a ser eliminados e deitados fora" (1). O DA é também considerado um fenómeno de uma sociedade consumista, já que muitos géneros alimentícios são de custo acessível e de fácil aquisição para a grande maioria de indivíduos nos países desenvolvidos (2).

A Organização Mundial da Saúde manifesta preocupação relativamente à promoção de hábitos alimentares saudáveis, especialmente em idade pediátrica, devido ao reconhecimento de que os hábitos e as escolhas alimentares poderão estar associados à saúde individual $(3,4)$. A par da promoção da saúde, a sustentabilidade alimentar apresenta-se como uma preocupação acrescida 
e atual $(5,6)$. Melhorar a qualidade da alimentação e reduzir o impacto ambiental torna-se uma preocupação global (7), pelo que as empresas prestadoras de serviços de restauração coletiva desempenham um papel fulcral no setor alimentar escolar (8).

Concomitantemente, a capitação (quantidade da medida de uma porção ou dose de um género alimentício usada em alimentação coletiva para a produção de refeições; quantidade de alimento per capita) (9), e a redução dos alimentos desperdiçados, desde a produção ao consumo, mostram-se essenciais para que o sistema alimentar global permaneça seguro (10). Neste sentido, a avaliação do DA tem sido uma medida indireta utilizada em contexto escolar para avaliar quer a satisfação com as refeições escolares, quer o seu contexto. Esta avaliação auxilia a direcionar, assertivamente, as modificações necessárias para uma maior credibilização e aceitação das refeições, conciliando aspetos essenciais à oferta alimentar, à otimização dos processos de gestão do serviço de alimentação, e integrando a refeição e o seu contexto.

\section{OBJETIVOS}

Avaliar o DA dos alunos que frequentam duas escolas públicas do distrito de Aveiro, na refeição do almoço, no que diz respeito ao prato, em duas unidades de restauração coletiva (URC) do setor escolar público.

\section{METODOLOGIA \\ População}

Foi avaliado o DA da refeição almoço de todos os alunos que frequentam os refeitórios da Escola A (5..$^{\circ}$ ao $12 .^{\circ}$ ano) e da Escola B (pré-escolar ao $9 .^{\circ}$ ano), com idades compreendidas entre os 3 e 19 anos.

\section{Amostra para a Avaliação do Desperdício Alimentar}

A amostra foi obtida por conveniência, e o DA analisado resultou de 2686 refeições servidas (RS), correspondestes a 10 dias de avaliação, 5 dias seguidos em cada escola.

\section{Material}

Para a avaliação do DA, utilizaram-se as sobras de produção e os restos dos pratos dos alunos das escolas supramencionadas. A definição destes conceitos está presente na Tabela 1.

\section{Metodologia}

Estudo descritivo observacional de desenho transversal.

O DA foi avaliado através do método de pesagem (Tabela 1). A recolha de informação foi efetuada pelo investigador principal, que pesou as sobras de produção, os restos do prato e os recipientes utilizados. Note-se que as cozinheiras serviam o prato de cada aluno, à exceção da componente hortícolas, na qual eram os alunos a colocar a quantidade a consumir.

Os procedimentos de quantificação do DA do prato foram os seguintes: Prato não composto, a pesagem total inicial e final realizou-se pesando separadamente os componentes do prato (conduto, guarnição e hortícolas); Prato composto, a pesagem total inicial e final realizou-se agregando todos os alimentos presentes $(9,11-12)$. Para garantir o cumprimento das boas práticas de higiene e segurança alimentar foi usado o seguinte material: bata, touca e luvas descartáveis; material de escritório; sacos de lixo e balança (marca Simão Vaz e modelo 200 A - balança mecânica industrial analógica com alcance de $150 \mathrm{~kg}$, capacidade mínima de 2,5 kg e precisão de +/- 0,050 kg).

Para determinação da sobra de produção, pesou-se a quantidade total de alimentos produzidos em cada item prato da refeição e, no final da sua distribuição aos alunos, os desperdiçados, sendo estes valores calculados pela diferença entre o valor final e o peso do recipiente vazio. Quando cada aluno terminava a refeição, os componentes que restavam no prato eram colocados em recipientes diferentes, um por cada componente, incluindo ossos, espinhas, cascas e peles, dado que foram inicialmente contabilizados na produção. Foi aplicada a parte edível referente ao "frango assado" e ao "red fish", de acordo com a tabela da composição dos alimentos portugueses (13). Por fim, procedeu-se ao cálculo das variáveis apresentadas na Tabela 1. Destaca-se o cálculo do número de alunos que poderiam ser alimentados com o DA obtido através das seguintes fórmulas: divisão do peso da sobra total pelo peso dos alimentos consumidos per capita e da divisão do peso do resto total pelo peso dos alimentos consumidos per capita.

O presente estudo decorreu durante 10 dias, 5 dias de recolha seguidos na escola A, seguindo-se 2 dias de intervalo e 5 dias de recolha seguidos na escola $B$. Este artigo encontra-se incluído num estudo original onde estavam também incluídos na avaliação do DA os itens da refeição sopa e sobremesa. No entanto, para efeitos de submissão, estes itens foram retirados.

Este projeto foi aprovado pela Comissão de Ética da Universidade do Porto (Parecer n. $75 /$ CEUP/2019). Os dados recolhidos foram inseridos e analisados no programa de tratamento de dados estatísticos SPSS ${ }^{\circledR}$ para Microsoft Windows ${ }^{\circledR}$ e no Excel $^{\circledR}$. A análise descritiva das variáveis foi efetuada a partir da determinação de medidas de tendência central. Para a associação das variáveis, testou-se a normalidade através do teste Shapiro-Wilk, utilizando-se os testes t Student e Mann-Whitney. O nível de significância considerado foi $5 \%(p<0,05)$.

\section{RESULTADOS}

\section{Distribuição da Amostra}

A ementa definida para o item prato foi sempre diferente, consistindo em 5 pratos de pescado e 5 de carne. Foram produzidas e servidas 2686 refeições, das quais 1110 (41\%) na URC da Escola A e 1576 (59\%) na URC da Escola B (Tabela 1). A média das RS, por dia, foi de 269, com uma média diária de 222 na URC da Escola A e de 315 na URC da Escola B. Das RS na URC da Escola B, 48\% são referentes ao pré-escolar e $1 .{ }^{\circ}$ ciclo.

Desperdício Alimentar do Item da Refeição Prato (conduto, guarnição, hortícolas)

Produziram-se um total de $465 \mathrm{~kg}$ de alimentos, dos quais $348 \mathrm{~kg}$ (75\%) foram consumidos e, dos $117 \mathrm{~kg}$ de DA (25\%), prevaleceram os restos em detrimento das sobras, $67 \%$ e $33 \%$ respetivamente (Tabela 1), perfazendo um índice de resto (IR) de 18\% e um índice de sobra (IS) de 8\%. As RS distribuíram-se por tipo de ementa: 1301 pratos de carne e 1385 de pescado.

\section{Desperdício Alimentar dos Componentes Conduto e Guarnição do} Item Prato

A URC da Escola B apresentou uma maior produção de alimentos (243,81 kg vs. 182,79 kg da URC da Escola A) e um IS e IR inferior (5,19\% e $16,52 \%$ vs. $8,00 \%$ e $21,61 \%$ da URC da Escola A, respetivamente (Tabela 2). A URC da Escola A produziu maior quantidade de alimentos per capita e apresentou um valor médio mais elevado de peso de resto per capita (PRpc), nomeadamente de 0,17 kg em relação a 0,16 kg da URC da Escola B. Contudo, ambas possuem o mesmo peso médio de sobra per capita (PSpc) $(0,01 \mathrm{~kg})$ - Tabela 2.

O IS foi, em média, de 6,89\%, exibindo um máximo de 14,74\% ("pescada gratinada com alecrim e arroz de ervilhas") na URC da Escola B e um mínimo de 0,00\% ("frango assado com massa" e "pá de porco 
Tabela 1

Metodologia para a quantificação do desperdício alimentar: legenda das variáveis calculadas e respetivos valores de distribuição total e por escola

\begin{tabular}{|c|c|c|c|c|c|}
\hline SIGLA & LEGENDA & DESCRIÇÃO & ESCOLA A & ESCOLA B & TOTAL \\
\hline RS & Refeições servidas & $\begin{array}{l}\text { Valor referente ao número de refeições servidas } \\
\text { (número efetivo de refeições consumidas pelos alunos), } \\
\text { correspondente ao número de alunos presentes diariamente } \\
\text { na URC do setor escolar. }\end{array}$ & 1110 & 1576 & 2686 \\
\hline $\mathrm{PS}(\mathrm{Kg})$ & Peso das sobras & $\begin{array}{l}\text { Pesagem dos alimentos crus e confecionados que não são } \\
\text { servidos. } \\
\text { - Peso total das sobras do conduto (carne, pescado, ovos, ...); } \\
\text { das sobras da guarnição (arroz, massa, batata, ...); das sobras } \\
\text { de hortícolas. }\end{array}$ & 18,37 & 20,55 & $\begin{array}{c}38,92 \\
(33 \%)^{\star}\end{array}$ \\
\hline $\mathrm{PR}(\mathrm{Kg})$ & Peso dos restos & $\begin{array}{l}\text { Pesagem de todos os alimentos que sobram nos pratos. } \\
\text { - Peso total dos restos do conduto (carne, pescado, ovos, ...); } \\
\text { dos restos da guarnição (arroz, massa, batata, ...); dos restos } \\
\text { de hortícolas. }\end{array}$ & 38,54 & 39,63 & $\begin{array}{l}78,17 \\
(67 \%)^{\star}\end{array}$ \\
\hline PAP (Kg) & Peso total dos alimentos produzidos & $\begin{array}{l}\text { Pesagem dos alimentos crus e confecionados prontos a } \\
\text { seguir para a linha de empratamento. }\end{array}$ & 199,24 & 265,31 & 464,55 \\
\hline PAD (Kg) & Peso total dos alimentos distribuídos & $\begin{array}{l}\text { Diferença entre o peso total de alimentos produzidos e o } \\
\text { peso total da sobra. }\end{array}$ & 180,87 & 244,76 & 425,63 \\
\hline PAPpc (Kg) & Peso dos alimentos produzidos per capita & $\begin{array}{l}\text { Divisão do peso dos alimentos produzidos pelo número de } \\
\text { refeições servidas. }\end{array}$ & 0,18 & 0,17 & 0,17 \\
\hline $\mathrm{PAC}(\mathrm{Kg})$ & Peso dos alimentos consumidos & $\begin{array}{l}\text { Diferença entre o peso dos alimentos distribuídos e o peso } \\
\text { do resto. }\end{array}$ & 142,33 & 205,12 & 347,46 \\
\hline $\mathrm{PACpc}(\mathrm{Kg})$ & Peso dos alimentos consumidos per capita & $\begin{array}{l}\text { Da divisão do peso dos alimentos consumidos pelo número } \\
\text { de refeições servidas. }\end{array}$ & 0,13 & 0,13 & 0,13 \\
\hline $\mathrm{PSpc}(\mathrm{Kg})$ & Peso de sobra per capita & Divisão do peso da sobra pelo número de refeições servidas. & 0,02 & 0,01 & 0,01 \\
\hline IS (\%) & Índice de sobra & $\begin{array}{l}\text { Divisão do peso da sobra pelo peso dos alimentos } \\
\text { produzidos. }\end{array}$ & 9 & 8 & 8 \\
\hline $\begin{array}{l}\text { AASA (n. } \\
\text { de alunos) }\end{array}$ & $\begin{array}{l}\text { Alunos que poderiam ser alimentados } \\
\text { com a sobra acumulada }\end{array}$ & $\begin{array}{l}\text { Divisão do peso da sobra total pelo peso dos alimentos } \\
\text { consumidos per capita. }\end{array}$ & 143,23 & 157,59 & 300,84 \\
\hline PRpc (Kg) & Peso do resto per capita & Divisão do peso do resto pelo número de refeições servidas. & 0,03 & 0,03 & 0,03 \\
\hline $\operatorname{IR}(\%)$ & Índice de resto & $\begin{array}{l}\text { Divisão do peso do resto pelo peso dos alimentos } \\
\text { distribuídos. }\end{array}$ & 21 & 16 & 18 \\
\hline $\begin{array}{l}\text { AARA (n. } .^{\circ} \\
\text { de alunos) }\end{array}$ & $\begin{array}{l}\text { Alunos que poderiam ser alimentados } \\
\text { com o resto acumulado }\end{array}$ & $\begin{array}{l}\text { Divisão do peso do resto total pelo peso dos alimentos } \\
\text { consumidos per capita. }\end{array}$ & 300,54 & 304,51 & 604,30 \\
\hline
\end{tabular}

*Distribuição do peso dos alimentos desperdiçados

\section{Tabela 2}

Distribuição relativa ao desperdício alimentar por componente do prato ("conduto e guarnição" vs. "hortícolas") e por escola vs. per capita

\begin{tabular}{|c|c|c|c|c|c|c|c|c|}
\hline \multicolumn{9}{|c|}{ COMPONENTE: CONDUTO E GUARNIÇÃO } \\
\hline & FREQUÊNCIA (n) & PAP (Kg) & PAD (Kg) & PAC (Kg) & PS (Kg) & PR (Kg) & IS (\%) & IR (\%) \\
\hline Escola A & 5 & 182,79 & 168,17 & 131,83 & 14,62 & 36,34 & 8,00 & 21,61 \\
\hline Escola B & 5 & 243,81 & 231,16 & 192,97 & 12,65 & 38,18 & 5,19 & 16,52 \\
\hline & \multicolumn{2}{|c|}{ FREQUÊNCIA $(n)$} & PAPpc (Kg) & \multicolumn{2}{|c|}{ PACpc (Kg) } & PSpc (Kg) & \multicolumn{2}{|c|}{$\operatorname{PRpc}(\mathrm{Kg})$} \\
\hline Escola A & \multicolumn{2}{|l|}{1110} & 0,17 & \multicolumn{2}{|c|}{0,12} & 0,01 & \multicolumn{2}{|c|}{0,03} \\
\hline Escola B & \multicolumn{2}{|l|}{1576} & 0,16 & \multicolumn{2}{|c|}{0,12} & 0,01 & \multicolumn{2}{|c|}{0,02} \\
\hline Total & \multicolumn{2}{|l|}{2686} & 0,16 & \multicolumn{2}{|c|}{0,12} & 0,01 & \multicolumn{2}{|c|}{0,03} \\
\hline
\end{tabular}

\begin{tabular}{|c|c|c|c|c|c|c|c|c|}
\hline \multicolumn{9}{|c|}{ COMPONENTE: HORTÍCOLAS } \\
\hline & FREQUÊNCIA (n) & $\operatorname{PAP}(\mathrm{Kg})$ & PAD $(K g)$ & $\operatorname{PAC}(K g)$ & PS (Kg) & PR (Kg) & IS (\%) & IR (\%) \\
\hline Escola A & 5 & 16,45 & 12,70 & 10,50 & 3,75 & 2,20 & 22,80 & 17,32 \\
\hline Escola B & 5 & 21,50 & 13,60 & 12,15 & 7,90 & 1,45 & 36,74 & 10,66 \\
\hline & \multicolumn{2}{|c|}{ FREQUÊNCIA (n) } & PAPpc (Kg) & \multicolumn{2}{|c|}{ PACpc (Kg) } & PSpc (Kg) & \multicolumn{2}{|c|}{ PRpc (Kg) } \\
\hline Escola A & \multicolumn{2}{|l|}{1110} & 0,02 & \multicolumn{2}{|c|}{0,01} & 0,00 & \multicolumn{2}{|c|}{0,00} \\
\hline Escola B & \multicolumn{2}{|l|}{1576} & 0,01 & \multicolumn{2}{|c|}{0,01} & 0,01 & \multicolumn{2}{|c|}{0,00} \\
\hline Total & \multicolumn{2}{|l|}{2686} & 0,01 & \multicolumn{2}{|c|}{0,01} & 0,00 & \multicolumn{2}{|c|}{0,00} \\
\hline
\end{tabular}

IR: Índice do resto

IS: Índice da sobra

PAC: Peso dos alimentos consumidos

PACpc: Peso dos alimentos consumidos per capita

PAD: Peso dos alimentos distribuídos

PAP: Peso dos alimentos produzidos

PAPpc: Peso dos alimentos produzidos per capita

PR: Peso dos restos
PRpc: Peso do resto per capita

PS: Peso das sobras

PSpc: Peso da sobra per capita 
estufada com cenouras e esparguete") na URC da Escola B, como apresentado na Tabela 3. Já a média de IR foi de 19,42\%, que resultou num valor médio por refeição de peso de resto (PR) de 7,45 kg. Das ementas analisadas, quatro apresentaram valores de IR superiores à média, nomeadamente: "red fish com salada de batata" (39,12\%), "pescada gratinada com alecrim e arroz de ervilhas" (27,48\%), "filetes de pescada com arroz e milho" (25,57\%) e "massa de carnes à lavrador" (19,98\%) - Tabela 3.

Desperdício Alimentar da Componente Hortícolas do Item Prato Verificou-se a oferta de 9 tipos de hortícolas distintos. Em média, por dia, produziram-se 3,80 kg de hortícolas, e distribuíram-se 2,63 kg, perfazendo $1,17 \mathrm{~kg}$ de sobras.

$\mathrm{Na}$ URC da Escola A foram produzidos $16,45 \mathrm{~kg}$ de hortícolas, apresentando um IS inferior (22,80\%) e um IR superior (17,32\%) em relação à URC da Escola $\mathrm{B}(21,50 \mathrm{~kg}, 36,74 \%$ e 10,66\% respetivamente). Analisando-se os dados per capita, apurou-se que a Escola A produz maior quantidade de hortícolas $(0,02 \mathrm{~kg}$ vs. 0,01 kg da Escola B) e um valor mais baixo de PSpc (0,00 vs. 0,01 da Escola B). Contudo, ambas possuem o mesmo PRpc (0,00 kg) - Tabela 2. O IS foi, em média, de 29,18\%, com um máximo foi 65,56\% ("alface, cenoura e couve-roxa") e um mínimo de 8,33\% ("pepino, tomate, ervilha e milho") na URC da Escola B (Tabela 3). Já os restos, perfizeram um total de 3,65 kg e, em média, cada refeição apresentou-se com 0,37 kg de resto. O IR dos hortícolas foi de, em média, 13,49\%, com um máximo de 22,86\% ("alface, beterraba e couve-roxa") na Escola A, e um mínimo de 3,23\% ("alface, cenoura e couve-roxa") na Escola B (Tabela 3).

Número de Alunos que Poderiam Ser Alimentados com o Desperdício Alimentar obtido

Concluiu-se que 300 alunos poderiam ser alimentados com a sobra existente: 143 na Escola A e 157 na Escola B. Dada a quantidade de alimentos consumidos per capita, 604 alunos poderiam ser alimentados com o resto: 300 na Escola A e 304 na Escola B. Assim, poderiam ser alimentados com o desperdício obtido 904 alunos no período de estudo (Tabela 1)

Tabela 3

Distribuição do desperdício alimentar de acordo com o item da refeição prato servido (componente conduto, guarnição e hortícolas)

\begin{tabular}{|c|c|c|c|c|c|c|c|c|c|c|}
\hline CONDUTO E GUARNIÇÃO & FREQ. $(n)$ & RS $(n)$ & PAP $(K g)$ & PAD (Kg) & PAC (Kg) & PR (Kg) & IR (\%) & PS (Kg) & IS (Kg) & DT $(\mathrm{Kg})$ \\
\hline $\begin{array}{l}\text { Pescada gratinada com alecrim e arroz de } \\
\text { ervilhas }\end{array}$ & 1 & 289 & 42,05 & 35,85 & 26,00 & 9,85 & 27,48 & 6,20 & 14,74 & 16,05 \\
\hline $\begin{array}{l}\text { Red fish com salada de batata (batata, } \\
\text { ervilha, feijão-verde e cenoura) }\end{array}$ & 1 & 239 & 34,34 & 31,92 & 19,43 & 12,49 & 39,12 & 2,42 & 7,04 & 14,90 \\
\hline Massa de carnes à lavrador & 1 & 213 & 49,70 & 44,55 & 35,65 & 8,90 & 19,98 & 5,15 & 10,36 & 14,05 \\
\hline Filetes de pescada com arroz e milho & 1 & 241 & 34,35 & 30,70 & 22,85 & 7,85 & 25,57 & 3,65 & 10,63 & 11,50 \\
\hline Douradinhos com arroz de tomate & 1 & 279 & 41,70 & 36,45 & 31,60 & 4,85 & 13,31 & 5,25 & 12,59 & 10,10 \\
\hline $\begin{array}{l}\text { Pá de porco estufada com cenouras e } \\
\text { esparguete }\end{array}$ & 1 & 332 & 64,75 & 64,75 & 56,05 & 8,70 & 13,44 & 0,00 & 0,00 & 8,70 \\
\hline Frango assado com massa & 1 & 339 & 46,26 & 46,26 & 38,17 & 8,08 & 17,48 & 0,00 & 0,00 & 8,08 \\
\hline Arroz de atum & 1 & 337 & 49,05 & 47,85 & 41,15 & 6,70 & 14,00 & 1,20 & 2,45 & 7,90 \\
\hline Carne à bolonhesa com massa fusili e cenouras & 1 & 263 & 39,95 & 38,15 & 34,00 & 4,15 & 10,88 & 1,80 & 4,51 & 5,95 \\
\hline Hambúrguers no forno com arroz de cenoura & 1 & 154 & 24,45 & 22,85 & 19,90 & 2,95 & 12,91 & 1,60 & 6,54 & 4,55 \\
\hline Total & 10 & 2686 & 426,60 & 399,33 & 324,81 & 74,52 & 18,66 & 27,27 & 6,39 & 101,79 \\
\hline DISTRIBUIÇÃO DO PAP: & & & & & $76 \%$ & $18 \%$ & & $6 \%$ & & \\
\hline Média & 1 & 269 & 42,66 & 39,93 & 32,48 & 7,45 & 19,42 & 2,73 & 6,89 & 10,18 \\
\hline Desvio-padrão & 0 & 60 & 10,90 & 11,61 & 11,25 & 2,86 & 8,90 & 2,23 & 5,17 & 3,87 \\
\hline Máximo & 1 & 339 & 64,75 & 64,75 & 56,05 & 12,49 & 39,12 & 6,20 & 14,74 & 16,05 \\
\hline Mínimo & 1 & 154 & 24,45 & 22,85 & 19,43 & 2,95 & 10,88 & 0,00 & 0,00 & 4,55 \\
\hline \multicolumn{11}{|l|}{ Hortícolas } \\
\hline Alface, cenoura, couve-roxa & 1 & 289 & 4,50 & 1,55 & 1,50 & 0,05 & 3,23 & 2,95 & 65,56 & 3,00 \\
\hline Alface, cenoura, couve-roxa & 1 & 239 & 4,95 & 2,85 & 2,40 & 0,45 & 15,79 & 2,10 & 42,42 & 2,55 \\
\hline Milho, cenoura e brócolos & 1 & 213 & 4,10 & 2,55 & 2,30 & 0,25 & 9,80 & 1,55 & 37,80 & 1,80 \\
\hline Alface, cenoura e milho & 1 & 241 & 4,30 & 3,05 & 2,60 & 0,45 & 14,75 & 1,25 & 29,07 & 1,70 \\
\hline Tomate e pepino & 1 & 279 & 3,80 & 2,50 & 2,25 & 0,25 & 10,00 & 1,30 & 34,21 & 1,55 \\
\hline Alface, milho e cenoura & 1 & 332 & 3,75 & 2,80 & 2,55 & 0,25 & 8,93 & 0,95 & 25,33 & 1,20 \\
\hline Alface, beterraba e couve-roxa & 1 & 339 & 3,85 & 3,50 & 2,70 & 0,80 & 22,86 & 0,35 & 9,09 & 1,15 \\
\hline Beterraba, alface, cenoura e couve-roxa & 1 & 337 & 2,00 & 1,40 & 1,15 & 0,25 & 17,86 & 0,60 & 30,00 & 0,85 \\
\hline Pepino, tomate, ervilha e milho & 1 & 263 & 4,20 & 3,85 & 3,40 & 0,45 & 11,69 & 0,35 & 8,33 & 0,80 \\
\hline Alface, tomate, pepino e couve-roxa & 1 & 154 & 2,50 & 2,25 & 1,80 & 0,45 & 20,00 & 0,25 & 10,00 & 0,70 \\
\hline Total & 10 & 2686 & 37,95 & 26,30 & 22,65 & 3,65 & 3,23 & 11,65 & 116,50 & 15,30 \\
\hline DISTRIBUIÇÃO DO PAP: & & & & & $60 \%$ & $9 \%$ & & $31 \%$ & & \\
\hline Média & 1 & 269 & 3,80 & 2,63 & 2,27 & 0,37 & 13,49 & 1,17 & 29,18 & 1,53 \\
\hline Desvio-padrão & 0 & 60 & 0,90 & 0,77 & 0,64 & 0,20 & 5,88 & 0,87 & 17,70 & 0,76 \\
\hline Máximo & 2 & 339 & 4,95 & 3,85 & 3,40 & 0,80 & 22,86 & 2,95 & 65,56 & 3,00 \\
\hline Mínimo & 1 & 154 & 2,00 & 1,40 & 1,15 & 0,05 & 3,23 & 0,25 & 8,33 & 0,70 \\
\hline $\begin{array}{l}\text { DT: Desperdício alimentar total } \\
\text { Freq.: Frequência } \\
\text { IR: Índice de resto } \\
\text { IS: Índice de sobra } \\
\text { PAC: Peso dos alimentos consumidos }\end{array}$ & & & & $\begin{array}{l}\text { PAD: Pe } \\
\text { PAP: Pe } \\
\text { PR: Pes } \\
\text { PS: Pesc } \\
\text { RS: Refe }\end{array}$ & $\begin{array}{l}\text { total dos ali } \\
\text { dos aliment } \\
\text { dos restos } \\
\text { das sobras } \\
\text { Ões servidas }\end{array}$ & $\begin{array}{l}\text { entos dist } \\
\text { produzid }\end{array}$ & dos & & & \\
\hline
\end{tabular}




\section{DISCUSSÃO DOS RESULTADOS}

Dada a relevância do DA para a sustentabilidade alimentar e ambiental (14-15), pretende-se contribuir para definir políticas que auxiliem a sua redução (16). O DA gerado neste estudo foi de $25 \%$, estando os restos do prato em maior quantidade (67\%), quando comparados com as sobras (33\%). No caso particular das sobras, poderão indicar um excesso de produção, quer por ineficiência da gestão da produção de refeições (17), quer por desadequação entre as recomendações alimentares, a capitação definida na legislação (18) e a quantidade consumida pelos alunos por motivos como o apetite, o ambiente do consumo, as características da refeição, entre outras, ou uma conjugação destes fatores.

O IS deste estudo foi de $8 \%$ na avaliação do DA global do prato e 6,9\% das suas componentes "conduto e guarnição" e 29,2\% da sua componente "hortícolas". Segundo alguns autores, valores de sobra superiores a 3\% são hipotéticos indicadores de uma má gestão da URC, sendo considerados inaceitáveis (19). No que se refere à componente do prato "conduto", verificou-se que quando o prato é composto, o IS mostrava-se mais elevado do que quando são diferenciados.

No que diz respeito aos restos, a literatura refere a sua associação direta com o grau de satisfação dos alunos (20), com a falta de consciencialização dos mesmos para o DA (21), assim como um desajuste entre o consumo real e a capitação exigida contratualmente (18). O IR geral foi de $18 \%$, com um valor médio para o item da refeição prato, de 19,4\% para os componentes "conduto e guarnição" e de $13,5 \%$ para o componente "hortícolas". No âmbito do DA em contexto escolar, não existem valores de referência nacionais para os restos do prato, tendo-se feito uso dos limites aceitáveis decretados pelo Conselho Federal de Nutricionistas Brasileiros, que menciona valores de desperdício alimentar de restos do prato inferiores a 10\% (22). Assim, todos os valores de IR encontrados foram superiores ao recomendado. Resultados idênticos, quer para as sobras, quer para os restos, foram encontrados na literatura (23-24). Note-se que os hortícolas foram a componente que apresentou maior IS e menor IR, o que indica que os alunos não o colocam tanto no prato, mas, quando o fazem, consomem o que colocam.

A URC da Escola A é a que manifesta maior quantidade de produção, sobras e restos (DA). Entre outros, tal pode ser fundamentado pelo empratamento das refeições em função de cada faixa etária como fator influenciador, pelo que a formação dos colaboradores deverá ser considerada. Para além disso, o facto de $48 \%$ das refeições da Escola B serem das valências até ao $1 .^{\circ}$ ciclo e, portanto, terem o acompanhamento por parte das educadoras no momento da refeição, pode contribuir para um menor DA (25). Um ambiente alimentar escolar saudável facilita o acesso a escolhas saudáveis e deve estar articulado com a criação de programas comunitários, envolvendo a URC, aliados a uma contínua educação alimentar de forma a promover a literacia alimentar e a reduzir o DA (26-28). Ademais, o estabelecimento de horários mais flexíveis para as refeições escolares também deveria ser considerado. Acresce o facto de, em idade pediátrica, a socialização com os colegas ser determinante para a intenção de finalizar a refeição rapidamente. Neste contexto, o acompanhamento das refeições por parte dos colaboradores da escola, é essencial para diminuir o DA, principalmente o IR (26).

Os resultados deste estudo apoiam os efeitos benéficos de análises holísticas e aprofundadas em intervenções para reduzir o DA, por forma a melhorar o consumo alimentar e promover uma adequada gestão e, consequentemente, levar a uma maior sustentabilidade do sistema de produção de refeições e à transição para uma economia circular dos refeitórios escolares.

No decurso do presente trabalho foi possível percecionar como principal limitação o facto das componentes "guarnição e conduto" terem sido quantificados de forma agregada, não permitindo distinguir o peso separado de cada elemento. Como pontos fortes é de realçar o facto da recolha de informação ter sido sempre realizada pelo investigador principal assim como a utilização de balança igual, o que facilitou a real comparação do peso dos alimentos nos resultados entre as URC. Foi também possível manter o normal funcionamento das URC.

\section{CONCLUSÕES}

O DA do prato observado neste estudo foi de $25 \%$ para o item da refeição prato, estando os restos do prato em maior quantidade, em relação às sobras de produção (67\% vs. $33 \%)$. Na avaliação do DA global do prato, o IS foi de $8 \%$ e o IR de $18 \%$, estando estes valores acima dos limites máximos recomendados. O IS é maior para a componente "hortícolas" $(29,2 \%)$ e o IR é maior para as componentes "conduto e guarnição" (19,4\%).

\section{DECLARAÇÃO DE INTERESSES}

Helena Ávila exerce a sua atividade profissional como diretora da qualidade de uma empresa de restauração coletiva e é docente convidada da licenciatura em Ciências da Nutrição de instituições do ensino superior privado.

\section{REFERÊNCIAS BIBLIOGRÁFICAS}

1. Parlamento Europeu. Como evitar o desperdício de alimentos: estratégias para melhorar a eficiência da cadeia alimentar na UE. In: Comissão da Agricultura e do Desenvolvimento Rural, editor. 2011.

2. Gjerris M, Gaiani S. Household food waste in Nordic countries: Estimations and ethical implications. Nordic Journal of Applied Ethics. 2013;7 (1), 6-23.

3. World Health Organization. Programme for Nutrition and Food Security WHO Regional Office for Europe. Food and nutrition policy for schools - A tool for the development of school nutrition programmes in the European Region. Copenhagen: WHO Regional Office for Europe. 2006.

4. World Health Organization. Taking Action on Childhood Obesity. 2018.

5. Food and Agriculture Organization of the United Nations. Methodological Proposal for Monitoring SDG Target 12.3 Sub-Indicator 12.3.1.A: The Food Loss Index Design, Data Collection Methods and Challenges.; 2019.

6. EAT-Lancet Commission. EAT-Lancet Commission brief for Food Service Professionals. Lancet; 2019.

7. Steen $\mathrm{H}$, Malefors $\mathrm{C}$, Roos E, Eriksson M. Identification and modelling of risk factors for food waste generation in school and pre-school catering units. Waste management (New York, NY). 2018;77:172-84.

8. Lassen AD, Christensen LM, Spooner MP, Trolle E. Characteristics of Canteens at Elementary Schools, Upper Secondary Schools and Workplaces that Comply with Food Service Guidelines and Have a Greater Focus on Food Waste. International journal of environmental research and public health. 2019;16(7).

9. Gomes S, Ávila H, Oliveira B, Franchini B. Capitações de Géneros Alimentícios para Refeições em Meio Escolar: Fundamentos, Consensos e Reflexões. Associação Portuguesa dos Nutricionistas, Faculdade de Ciências da Nutrição e Alimentação da Universidade do Porto, Programa Nacional para a Promoção da Alimentação Saúdavel da Direção-Geral da Saúde. editor. Porto. 2015.

10. Willett W, Rockström J, Loken B, Springmann M, Lang T, Vermeulen S, et al. Food in the Anthropocene: the EAT-Lancet Commission on healthy diets from sustainable food systems. The Lancet. 2019;393(10170):447-92.

11. Chapman LE, Richardson S, McLeod L, Rimm E, Cohen J. Pilot Evaluation of Aggregate Plate Waste as a Measure of Students School Lunch Consumption. Journal of the Academy of Nutrition and Dietetics. 2019. 
12. Pinto RS, Pinto RMS, Melo FFS, Campos SS, Cordovil CM. A simple awareness campaign to promote food waste reduction in a University canteen. Waste management (New York, NY). 2018;76:28-38.

13. Instituto Nacional de Saúde Dr. Ricardo Jorge. Tabela da Composição de Alimentos. 2006.

14. Mota EBF, Bezerra IWL, Seabra IMJ, Silva GCB, Rolim PM. Metodologia de avaliação de cardápio sustentável para serviços de alimentação. HOLOS. 2017. 4:381-394.

15. Derqui B, Fernandez V, Fayos T. Towards more sustainable food systems. Addressing food waste at school canteens. Appetite. 2018;129:1-11.

16. Comissão Nacional de Combate ao Desperdício Alimentar. Iniciativas de Combate ao Desperdício Alimentar. Programa de gestão de resíduos alimentares nas unidades dos SASUM2019.

17. Ribeiro J, Rocha A. Impacto Económico do Desperdício Alimentar num centro escolar. Acta Portuguesa de Nutrição. 2019. 19: 36-41.

18. Ministério da Educação - Direção-Geral da Educação. Circular n³097/DGE/2018. 2018-08-08) acedido a 02/02/2021, disponível em https://www.dge.mec.pt/sites/ default/files/Esaude/circular2brefeitorios2bjulho2b2018.pdf.

19. Vaz C. Restaurantes: controlando custos e aumentando lucros. Brasília: LGE; 2006. 20. Ferigollo MC, Busato MA. Desperdício de alimentos em unidades de alimentação e nutrição: Uma revisão integrativa da literatura. HOLOS. 2018. 01: 91-102.

21. Machado CCB, Mendes CK, Souza PG, Martins KSR, Silva KCC. Avaliação do índice de resto ingesta de uma unidade de alimentação e nutrição institucional de Anápolis-Go. Ensaios e Ciência: Ciências Biológicas, Agrágrias e da Saúde. 2012. 16: 151-162.

22. Conselho Federal de Nutricionistas. Resolução CFN N 380/2005. Brasil. 2005. 23. Oliveira D, Liz Martins M, Fonseca L, Rocha A. Food waste index as an indicator of menu adequacy and acceptability in a Portuguese Mental Health Hospital. Acta Portuguesa de Nutrição 20. 2020; 14-18.

24. Rabelo N, Alves T. Avaliação do percentual de resto-ingestão e sobra alimentar em uma unidade de alimentação e nutrição institucional. Revista Brasileira de Tecnologia Agroindustrial. 2016;10.

25. Martins M, Rodrigues S, Cunha L, Rocha A. Strategies to reduce plate waste in primary schools - experimental evaluation. Public Health Nutrition. 2015;19 (8):1517-25. 26. Araujo L. Rocha A. Avaliação e controlo do desperdício alimentar em refeitórios escolares do município de barcelos. Acta Portuguesa de Nutrição. 2017. 8: 6-9.

27. Centers for Disease Control and Prevention. Comprehensive Framework for Addressing the School Nutrition Environment and Services. 2019.

28. Quinn EL, Johnson DB, Podrabsky M, Saelens BE, Bignell W, Krieger J. Effects of a Behavioral Economics Intervention on Food Choice and Food Consumption in Middle-School and High-School Cafeterias. Preventing chronic disease. 2018; 15:E91. 